\title{
miR-34a: Multiple Opposing Targets and One Destiny in Hepatocellular Carcinoma
}

\author{
Radwa Alaa Yacoub ${ }^{1}$, Injie Omar Fawzy², Reem Amr Assal ${ }^{2}$, Karim Adel Hosny ${ }^{3}$, \\ Abdel-Rahman Nabawy Zekri ${ }^{4}$, Gamal Esmat ${ }^{5}$, Hend Mohamed El Tayebi ${ }^{2}$ \\ and Ahmed Ihab Abdelaziz*2,6
}

\begin{abstract}
${ }^{1}$ Pharmaceutical Biology Department, Faculty of Pharmacy and Biotechnology, German University in Cairo, Cairo, Egypt; ${ }^{2}$ Pharmacology and Toxicology Department, Faculty of Pharmacy and Biotechnology, German University in Cairo, Cairo, Egypt; ${ }^{3}$ Department of General Surgery, Faculty of Medicine, Cairo University, Cairo, Egypt; ${ }^{4}$ Virology and Immunology, Cancer Biology Department, National Cancer Institute, Cairo University, Cairo, Egypt; ${ }^{5}$ Department of Endemic Medicine and Hepatology, Cairo University, Cairo, Egypt; ${ }^{6}$ School of Medicine, New Giza University, Cairo, Egypt
\end{abstract}

\begin{abstract}
Background and Aims: The role of miR-34a in hepatocellular carcinoma (HCC) is controversial and several unresolved issues remain, including its expression pattern and relevance to tumor etiology, tumor stage and prognosis, and finally, its impact on apoptosis. Methods: miR-34a expression was assessed in hepatitis $C$ virus (HCV)-induced non-metastatic HCC tissues by RT-Q-PCR. Huh-7 cells were transfected with miR-34a mimics and the impact of miR-34a was examined on 84 pro-apoptotic/anti-apoptotic genes using PCR array; its net effect was tested on cell viability via MTT assay. Results: miR34 a expression was up-regulated in HCC tissues. Moreover, miR-34a induced a large set of pro-apoptotic/anti-apoptotic genes, with a net result of triggering apoptosis and repressing cell viability. Conclusions: HCC-related differential expression of miR-34a could be etiology-based or stage-specific, and low expression of miR-34a may predict poor prognosis. This study's findings also emphasize the role of miR-34a in apoptosis.

(C) 2016 The Second Affiliated Hospital of Chongqing Medical University. Published by XIA \& HE Publishing Inc. All rights reserved.
\end{abstract}

\section{Introduction}

microRNA (miR)-34a has emerged as an important miR in comprehensive research studies, and numerous studies have proposed its considerable impact on cell proliferation and survival. ${ }^{1}$ miR-34a has several experimentally validated downstream target genes involved in cell cycle regulation, including NMYC, ${ }^{2}$ CCND $1,{ }^{3}$ CCNE2, CDK4, CDK6, ${ }^{4}$ and C-MET. ${ }^{4,5}$ These genes promote cells to proceed through G1 phase into $S$ phase via the Rb-E2F signaling pathway, and

Keywords: MicroRNA; Hepatocellular carcinoma; Apoptosis; PCR array.

Abbreviations: miRNA or miR, microRNA; HCC, hepatocellular carcinoma; HCV, hepatitis $\mathrm{C}$ virus; $\mathrm{HBV}$, hepatitis $\mathrm{B}$ virus.

Received: 24 August 2016; Revised: 29 November 2016; Accepted: 22 December 2016

DOI: $10.14218 /$ JCTH.2016.00031.

* Correspondence to: Ahmed Ihab Abdelaziz, School of Medicine, Health Sciences Research Center (HSRC), New Giza University (NGU), Office: 2.26, New Giza km 22 Cairo-Alex desert road, Cairo 11835, Egypt. Tel: +20-2-38277847, E-mail: aihab@ ngu.edu.eg ectopic expression of miR-34a was shown to induce cell cycle arrest in the G1 phase. ${ }^{6}$ Moreover, it was reported that overexpression of miR-34a could down-regulate CDC25C and phosphorylated CDC2 expression, which in turn inhibits cell proliferation via cell cycle G2 phase arrest. ${ }^{7,8}$ miR-34a was reported to directly target $Y Y 1$, a negative regulator of the tumor suppressor $\mathrm{p} 53,{ }^{9}$ and induce apoptosis in neuroblastoma cell lines. ${ }^{10}$ Furthermore, miR-34a ectopic expression in breast cancer cells suppressed cell proliferation and invasion, and induced apoptosis, and miR-34a expression was found to be inversely correlated to BCL-2 and SIRT1 expression. ${ }^{11}$

In hepatocellular carcinoma (HCC), two independent studies reported that miR-34a had no impact on apoptosis. ${ }^{5,12}$ On the contrary, another research group showed that miR-34a increased caspase- 3 and -7 activity and induced apoptosis. ${ }^{13}$ Thus, the role of miR-34a in apoptosis in HCC is ambiguous. Another controversial aspect of miR-34a regards its pattern of expression and its relevance to the tumor etiology and stage. miR-34a was found to exhibit a different pattern of expression in hepatitis $\mathrm{C}$ virus (HCV)-related $\mathrm{HCC}^{14}$ than in hepatitis $\mathrm{B}$ virus (HBV)-induced HCC. 5,15 In addition, the expression level of miR-34a was found to vary between HCC tissues in clinical TNM stages III and IV as compared to that in stages I and II. ${ }^{13}$ It was also reported that metastatic HCC tissues showed a significantly different miR-34a expression profile compared to non-metastatic tissues. ${ }^{5,13}$ Despite these controversies, miR-34a has entered phase I clinical trials for liver cancer. ${ }^{16}$

Thus, in this study, we first aimed to elucidate the discrepancies regarding miR-34a expression pattern via investigating miR-34a expression in a cohort of patients with HCVprogressed non-metastatic HCC. Then, we aimed at dissecting the potentially related apoptotic pathways through investigating the impact of miR-34a on several downstream apoptotic and anti-apoptotic genes in HCC. Finally, we assessed the net impact of miR-34a on cell viability.

\section{Methods}

\section{Study patients}

This study comprised 22 HCC patients who underwent liver transplant surgery at the Kasr El Ainy Hospital, Cairo 
University, Egypt. In addition, 19 healthy liver biopsies were obtained. The healthy liver tissues were obtained from liver donors who volunteered for lobar transplantation surgery for HCC patients. Healthy livers represented non-cirrhotic tissues, and all donors were negative for HCV and HBV infection. Written consent, from both the patient and the donor, to take liver tissues during the transplantation was obtained prior to the surgery. Patients were subjected to clinical assessment (results presented in Table 1). HCC was diagnosed in all patients by examining the stage and grade of the tumor. Liver function enzymes (aspartate aminotransferase, alanine aminotransferase, and alkaline phosphatase) and albumin levels in serum were measured. Also the presence of hepatotropic infections, such as presence of HCV or HBV, was checked. All patients were confirmed as non-metastatic with no extra-hepatic manifestations and no vascular invasion, in order to comply with the expanded Milan criteria (UCSF criteria) for liver transplantation (i.e. single tumor of $<6.5 \mathrm{~cm}$ in diameter, or 2 or 3 tumors, each of $\leq 4.5 \mathrm{~cm}$ in diameter or with total tumor diameter $\leq 8 \mathrm{~cm}$ ) as shown in Table 2 . The majority of the patients $(63.6 \%)$ had more than one focal lesion, as indicated in the pathology report. All experiments were performed in compliance with the guidelines of the Institutional Review Board of Kasr El Ainy Medical School in Cairo University and the ethical committee of the German University in Cairo and in accordance to the ethical standards of the Declaration of Helsinki.

\section{Cell culture}

Huh-7 cells were maintained in Dulbecco's modified Eagle's medium (DMEM) (Lonza, Switzerland) supplemented with $4.5 \mathrm{~g} / \mathrm{L}$ glucose, $4 \mathrm{mmol} / \mathrm{L}$ L-glutamine, $10 \%$ fetal bovine serum, $1 \%$ penicillin/streptomycin and Mycozap (1:500; Lonza) at $37^{\circ} \mathrm{C}$ in $5 \% \mathrm{CO}_{2}$ atmosphere.

Table 1. Clinical characteristics of the 22 hepatocellular carcinoma (HCC) patients

\begin{tabular}{ll}
\hline \begin{tabular}{l} 
Age: mean \\
\multicolumn{1}{c}{ range }
\end{tabular} & $\begin{array}{l}49 \pm 13.5 \\
35.5-62.5\end{array}$ \\
$\begin{array}{l}\text { Sex: male/female } \\
\begin{array}{l}\text { Aspartate } \\
\text { aminotransferase (U/L) }\end{array}\end{array}$ & $21 / 1$ \\
$\begin{array}{l}\text { Alanine aminotransferase } \\
\text { (U/L) }\end{array}$ & $100.5 \pm 65.8$ \\
$\begin{array}{l}\text { Alkaline Phosphatase (U/L) } \\
\text { Serum albumin (g/dL) }\end{array}$ & $110.2 \pm 60.7$ \\
$\begin{array}{l}\text { Serum alpha-fetoprotein } \\
\text { (ng/mL) }\end{array}$ & $4.6 \pm 1.5$ \\
HCV status & $155.7 \pm 22.3$ \\
HBV status &
\end{tabular}

${ }^{a}$ HCV status was determined using anti-HCV antibody and/or HCV viral RNA quantification.

${ }^{\mathrm{b}} \mathrm{HBV}$ status was determined using anti-HBc and anti-HBs antibodies and by detection of HBsAg.
Table 2. Number/sizes of focal lesions according to expanded Milan criteria

\begin{tabular}{|c|c|c|}
\hline Patients & $\begin{array}{l}\text { Number of } \\
\text { focal lesions }\end{array}$ & Size of focal lesions \\
\hline Patient 1 & Unifocal & $2.5 \mathrm{~cm}$ \\
\hline Patient 2 & 3 focal lesions & $2 \mathrm{~cm}, 2.5 \mathrm{~cm}$ and $3 \mathrm{~cm}$ \\
\hline Patient 3 & 3 focal lesions & $2 \mathrm{~cm}, 2 \mathrm{~cm}$ and $3.5 \mathrm{~cm}$ \\
\hline Patient 4 & Unifocal & $1.5 \times 2 \mathrm{~cm}$ \\
\hline Patient 5 & 3 focal lesions & $3 \times 4 \mathrm{~cm}, 1 \mathrm{~cm}$ and $1 \mathrm{~cm}$ \\
\hline Patient 6 & Unifocal & $4 \mathrm{~cm}$ \\
\hline Patient 7 & 3 focal lesions & $4 \mathrm{~cm}, 1 \mathrm{~cm}$ and $1 \mathrm{~cm}$ \\
\hline Patient 8 & 3 focal lesions & $1 \mathrm{~cm}, 1 \mathrm{~cm}$ and $1.5 \mathrm{~cm}$ \\
\hline Patient 9 & Unifocal & $2.5 \mathrm{~cm}$ \\
\hline Patient 10 & 2 focal lesions & $1 \mathrm{~cm}$ and $1.7 \mathrm{~cm}$ \\
\hline Patient 11 & 3 focal lesions & $1 \mathrm{~cm}$ each \\
\hline Patient 12 & Unifocal & $3 \mathrm{~cm}$ \\
\hline Patient 13 & 3 focal lesions & $3 \mathrm{~cm}, 1.5 \mathrm{~cm}$ and $2 \mathrm{~cm}$ \\
\hline Patient 14 & 3 focal lesions & $1 \mathrm{~cm}, 1 \mathrm{~cm}$ and $4 \mathrm{~cm}$ \\
\hline Patient 15 & 2 focal lesions & $3 \mathrm{~cm}$ and $1.5 \mathrm{~cm}$ \\
\hline Patient 16 & 2 focal lesions & $1.5 \mathrm{~cm}$ and $3 \mathrm{~cm}$ \\
\hline Patient 17 & 3 focal lesions & $2.5 \mathrm{~cm}, 2.5 \mathrm{~cm}$ and $1.5 \mathrm{~cm}$ \\
\hline Patient 18 & 3 focal lesions & $1.5 \mathrm{~cm}, 1 \mathrm{~cm}$ and $1 \mathrm{~cm}$ \\
\hline Patient 19 & Unifocal & $2 \mathrm{~cm}$ \\
\hline Patient 20 & Unifocal & $1.5 \mathrm{~cm}$ \\
\hline Patient 21 & 3 focal lesions & $3 \mathrm{~cm}, 2.5 \mathrm{~cm}$ and $1 \mathrm{~cm}$ \\
\hline Patient 22 & Unifocal & $3 \mathrm{~cm}$ \\
\hline
\end{tabular}

\section{Transfection of miRNA oligonucleotides}

Huh-7 cell lines were transfected with miR-34a mimics (Catalog \#MSY0000255; Qiagen, Germany). Transfection experiments were carried out in triplicate, using the HiPerfect Transfection Reagent (Qiagen) according to the manufacturer's protocol which called for $37.5 \mathrm{ng}$ oligonucleotides for Huh-7 cells transfected in a 24-well plate; the experiment was repeated 3 times. Cells that were only exposed to transfection reagent are designated as Mock cells, while cells transfected with miR-34a mimics are designated as miR-34a cells.

\section{mRNA and miRNA extraction}

Total cellular mRNA and miRNA were extracted from the liver biopsies. Fresh liver samples (HCC and healthy tissues) were collected during surgery and snap-frozen immediately in liquid nitrogen. The specimens were then manually pulverized in liquid nitrogen and approximately $100 \mathrm{mg}$ of tissue powder was used for large and small RNA extraction with the mirVana miRNA Isolation Kit (Ambion, USA), according to the manufacturer's protocol.

For extraction of total cellular mRNA from cultured cells, Huh-7 cells were harvested 48 hours post-transfection and lysed using Bizol Reagent (Invitrogen). Chloroform was added and the aqueous phase containing the RNA was collected. RNA was precipitated using isopropyl alcohol. The RNA pellet 
Yacoub R. et al: miR-34a in HCC

was then washed using $75 \%$ ethanol and dissolved in DPECtreated water.

\section{RNA concentration and integrity}

RNA concentration was measured using a NanoDrop spectrophotometer (Thermo Fisher Scientific, USA) and was found to be $>500 \mathrm{ng} / \mu \mathrm{L}$. In addition, RNA integrity was confirmed by 18S rRNA band detection on $1 \%$ agarose gel electrophoresis. RNA samples with optical density $(260 / 280)$ of $>2$ were excluded from the study.

\section{miRNA and mRNA quantification by TaqMan Real-Time Q-PCR}

The miRNA extracted from liver tissues was reverse transcribed into single-stranded cDNA using the TaqMan ${ }^{\circledR}$ MicroRNA Reverse Transcription Kit (ABI, USA) with specific primers for hsa-miR-34a and RNU6B. Relative expression of miR-34a and RNU6B (internal control for normalization) was quantified using TaqMan Real-Time Q-PCR (ABI Assay IDs: 000426 and 001093, respectively) with the StepOne ${ }^{\mathrm{TM}}$ Real-Time PCR System (ABI). Relative expression was calculated using the $2^{-\Delta \Delta C T}$ method. All PCR reactions, including controls, were run in duplicate.

\section{PCR array}

Total RNA extracted from Huh-7 cells was reverse transcribed into cDNA using the RT2 First-Strand Kit (SA Biosciences, Germany). The CDNA was then combined with RT2 SYBR Green qPCR Master Mix (SA Biosciences) and equal aliquots of this mixture were added to 96-well plates of the Human Apoptosis RT2 Profiler PCR Array (SA Biosciences) that contained the gene-specific primers, human ATGs PAHSO12z (SA Biosciences). PCR was performed using the 7500 Fast Real-Time PCR System (ABI) with the following thermal cycling conditions: $15 \mathrm{~min}$ at $95^{\circ} \mathrm{C}$ for 1 cycle, $10 \mathrm{~s}$ at $95^{\circ} \mathrm{C}$, and $1 \mathrm{~min}$ at $60^{\circ} \mathrm{C}$ for 40 cycles. Expression of 84 key genes involved in apoptosis and 5 housekeeping genes (B2M, ACTB, GAPDH, HPRT1 and RPLPO) was profiled. Threshold cycle data obtained was analyzed using the RT2 Profiler Software (version 3.5; SA Biosciences). Relative gene expression was calculated using the $2^{-\Delta \Delta \mathrm{CT}}$ method.

\section{Cell viability assay (MTT)}

For the MTT (3-(4,5-dimethylthiazol-2-yl)-2,5-diphenyltetrazolium bromide) assay, 160,000 Huh-7 cells were seeded in $500 \mu \mathrm{L}$ of media per well in a 24-well plate and incubated for 24 hours prior to transfection with $37.5 \mathrm{ng}$ oligonucleotides (according to the HiPerfect protocol). Forty-eight hours posttransfection, $1 \mathrm{~mL}$ of MTT solution ( $5 \mathrm{mg} / \mathrm{mL} \mathrm{MTT}$ in $10 \%$ PBS) was added to each well. After incubation for 5 hours, formazan (MTT metabolic product) was resuspended in $1 \mathrm{~mL}$ of lysis solution (DMSO: absolute ethanol). Then, $200 \mu \mathrm{L}$ from each well was transferred to a 96-well plate and colorimetric measurements and absorbances (572 nm) were measured using the Wallac 1420 Victor2 Multilabel Counter (Perkin Elmer, USA). To eliminate any intensifying absorption that the culture medium itself could cause, culture medium without cells was assayed with the MTT solution and the subsequent reading was subtracted from all other values. The experiment was performed in triplicate and repeated three times.

\section{Statistical analysis}

All data are presented as mean with standard error of the mean (SEM). GraphPad Prism 5.0 was used to prepare all figures. Statistical significance of the data was analyzed using unpaired Student's $t$-test, and determined at a $p$ value of $<0.05(* p<0.05, * * p<0.01, * * * p<0.001)$.

\section{Results}

\section{miR-34a screening in HCV-induced non-metastatic HCC liver tissues}

The pattern of expression of miR-34a was investigated in HCC tissues taken from 22 patients with HCV-induced nonmetastatic HCC and in 19 liver tissues obtained from healthy donors undergoing liver transplantation. The relative expression of miR-34a was significantly higher in HCC tissues compared to healthy tissues ( $p=0.02^{*}$ ) (Fig. 1 ).

\section{Effect of miR-34a on a set of pro- and anti-apoptotic genes involved in apoptosis}

In order to investigate the impact of miR-34a on apoptosis, Huh-7 cells were transfected with miR-34a mimics, and effect of miR-34a ectopic overexpression was observed on the expression of 84 genes with critical roles in apoptosis. PCR array revealed that forcing the expression of miR-34a in Huh-7 cells resulted in altered gene expression of a set of proapoptotic/anti-apoptotic genes. Specifically, it up-regulated the expression of 26 pro-apoptotic genes (Fig. 2a) and 10 anti-apoptotic genes (Fig. 2c); in addition, it repressed the expression of 17 pro-apoptotic genes (Fig. 2b) as well as 8 anti-apoptotic genes (Fig. $2 \mathrm{~d}$ ), while the remaining genes were unaffected.

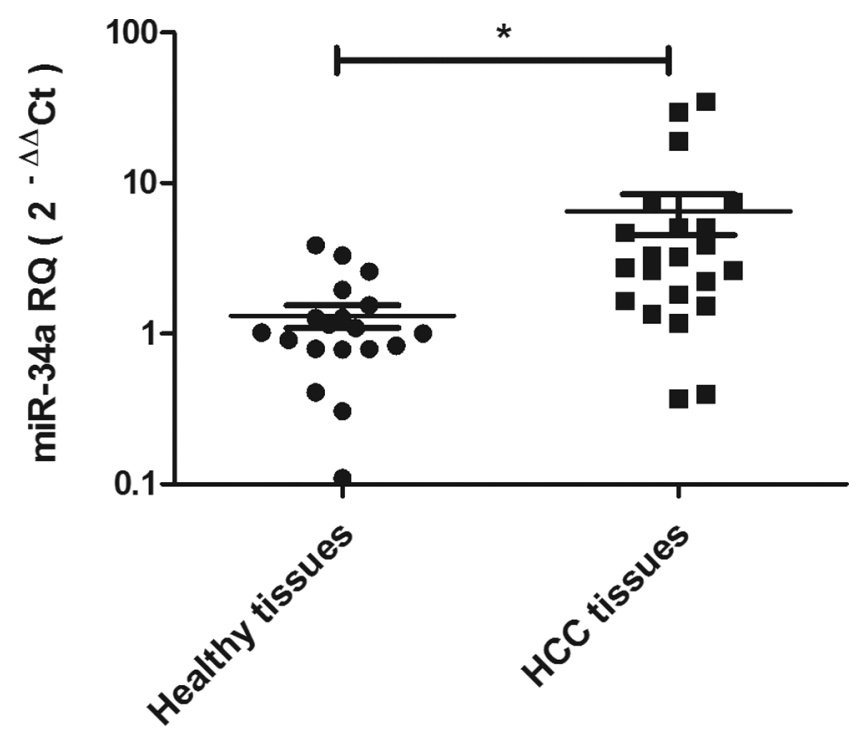

Fig. 1. Expression pattern of miR-34a in HCV-induced non-metastatic HCC tissues. miR-34a expression pattern was investigated using RT-Q-PCR in liver tissues obtained from 22 patients with HCV-induced non-metastatic $\mathrm{HCC}$ and 19 healthy donors. miR-34a showed significantly increased expression in HCC tissues, compared to healthy liver tissues. Expression of miR-34a was normalized, in each sample, to the housekeeping gene RNU6B. Results were analyzed using unpaired Student's $t$-test where $p<0.05^{*}, p<0.01 * *, p<0.001 * * *$. 

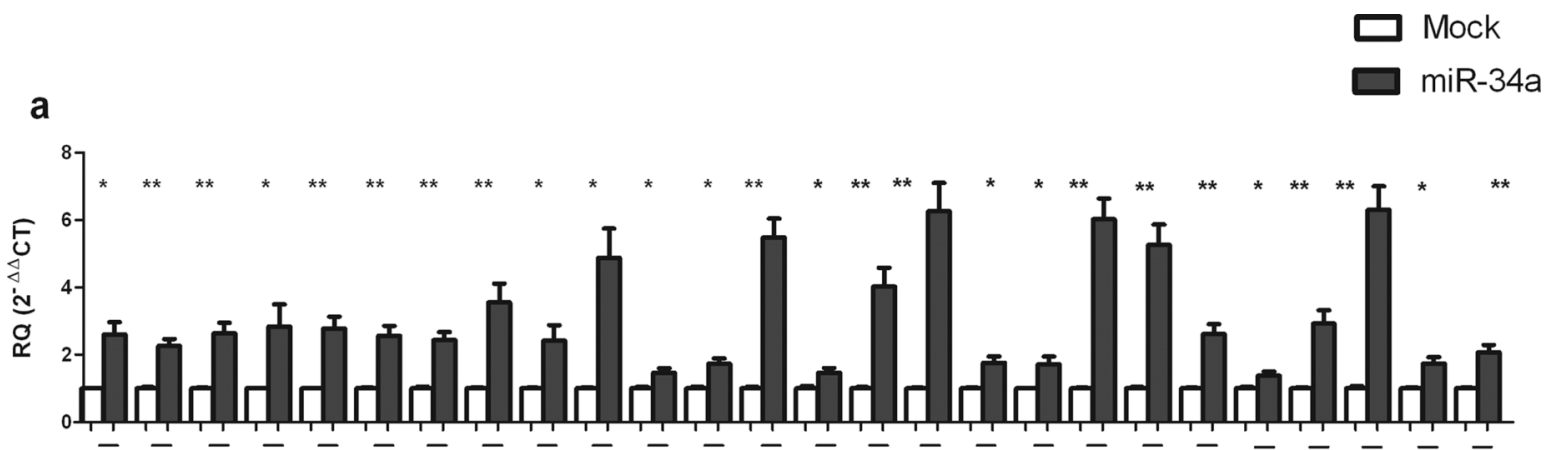

b

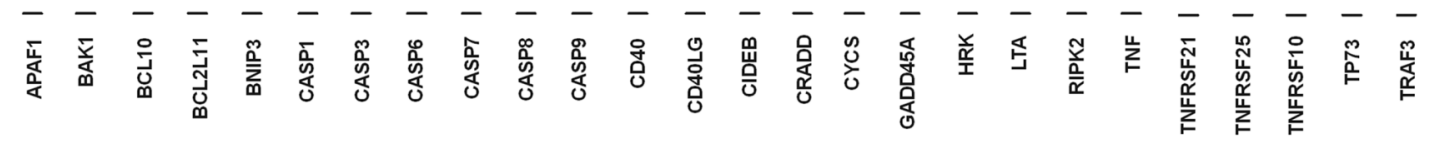
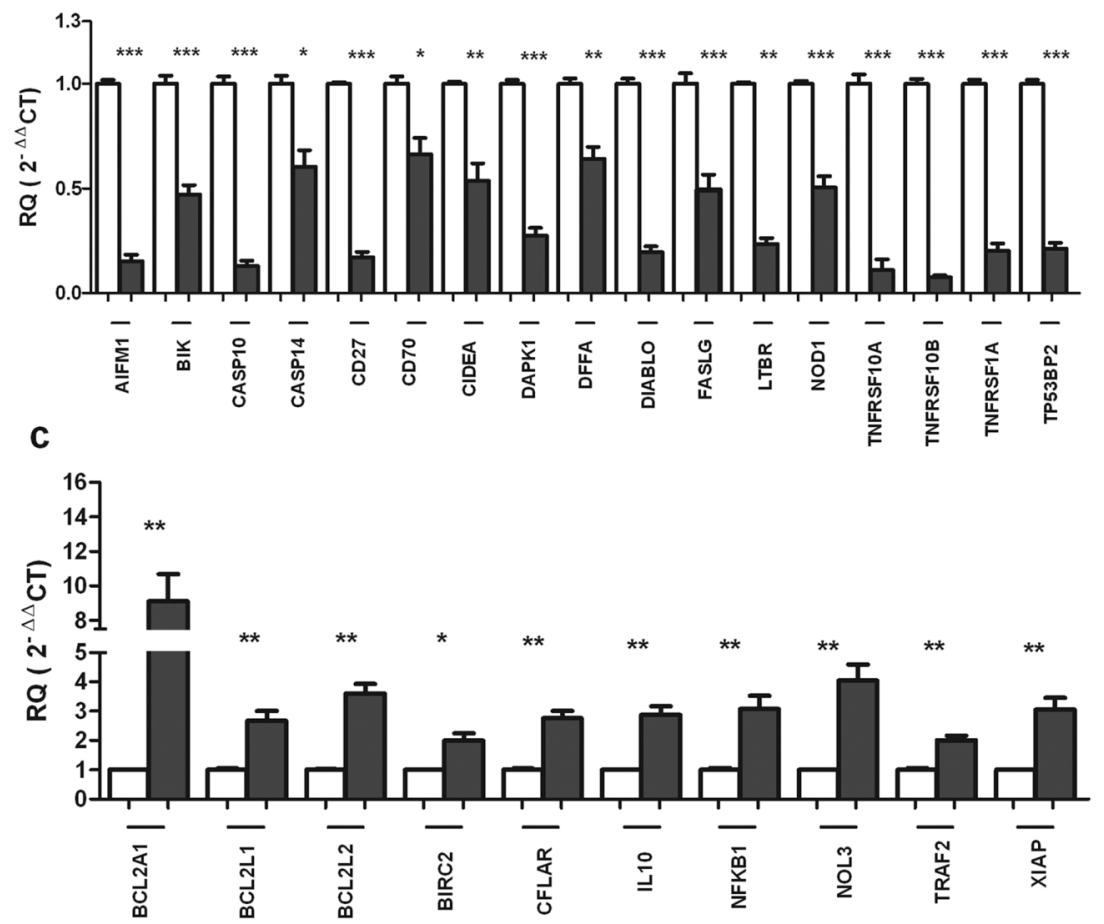

d

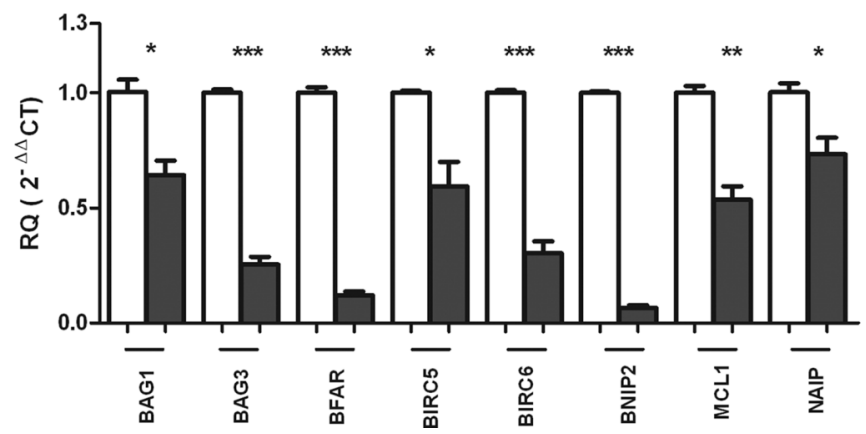

Fig. 2. Impact of miR-34a on the expression of pro-apoptotic and anti-apoptotic genes. Huh-7 cells were transfected with miR-34a mimics, and the expression of several apoptosis-relevant genes was evaluated using PCR array in transfected cells as compared to Mock (untransfected) cells. (a) Ectopic overexpression of miR-34a upregulated the expression of 26 pro-apoptotic genes (b) and down-regulated the expression of 17 pro-apoptotic genes. (c) Expression of 10 anti-apoptotic genes was induced, (d) while expression of 8 anti-apoptotic genes was repressed. Experiments were performed in triplicate and repeated 3 times. Expression of each gene was normalized to 5 housekeeping genes (B2M, ACTB, GAPDH, HPRT1 and RPLPO). Each bar represents the mean \pm SEM, and results were analyzed using unpaired Student's $t$-test where $p<0.05^{*}, p<0.01^{* *}, p<0.001^{* * *}$. 


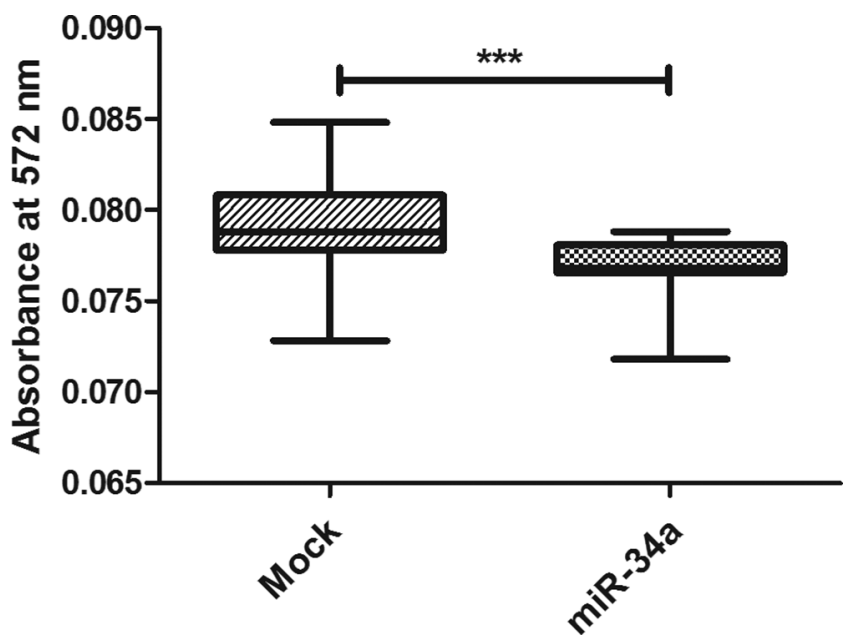

Fig. 3. Impact of miR-34a on cell viability (MTT assay). miR-34a mimics remarkably inhibited cellular growth and viability in Huh-7 cells. Experiments were performed in triplicate and repeated 3 times. Absorbance was measured at $572 \mathrm{~nm}$. Each bar represents the mean \pm SEM, and results were analyzed using unpaired Student's $t$-test where $p<0.05 *, p<0.01 * *, p<0.001 * * *$.

\section{Impact of miR-34a on cell viability}

MTT assay was used for investigating cell viability. Forced expression of miR-34a in the Huh-7 cell line showed marked repression of cellular viability $(p=0.0006 * * *)$ as compared to the Mock cells (Fig. 3).

\section{Discussion}

miR-34a is a crucial miRNA that has been investigated in cancers and also subject to clinical trials. miR-34a has been investigated in $\mathrm{HCC}$, yielding controversial results and raising several questions that encompass diverse aspects: first, the expression pattern of miR-34a and its relevance to the tumor etiology; second, the relevance of its expression pattern to the tumor stage and prognosis; and finally, its impact on apoptosis.

In order to answer the first question that has been raised regarding the pattern of miR-34a expression and its association with the tumor etiology, we focused on HCV-induced HCC. In this study, miR-34a expression was shown to be upregulated in $\mathrm{HCV}$-induced $\mathrm{HCC}$ tissues (Fig. 1). This finding coincides with previous studies showing that miR-34a was up-regulated in HCV-related HCC, compared to normal liver tissues. ${ }^{14,17}$ On the contrary, miR-34a expression was found to be down-regulated in HBV-positive HCC tissues. ${ }^{5,13,15,18}$ In light of these findings, it may be presumed that the miR-34a expression pattern might depend on the etiology of HCC.

Another imperative question was the correlation between miR-34a expression pattern and the prognosis of HCC. In this study, results showed that miR-34a expression was up-regulated in HCV-induced non-metastatic HCC tissues (Fig. 1). In a previous study, miR-34a was found to be overexpressed in non-metastatic tissues, compared to metastatic ones, and in clinical TNM stages I and II, compared to stages III and IV. ${ }^{5,13}$ Taken together, these findings suggest that the miR-34a expression pattern might be stage-specific and miR-34a low expression may predict poor prognosis.
In an attempt to unveil the role of miR-34a in apoptosis, providing an answer for the third inquiry, the expression of miR-34a was forced in Huh-7 cells, and by using PCR array the expression of a set of 84 pro-apoptotic and anti-apoptotic genes was investigated. Interestingly, the expression of 26 pro-apoptotic genes was found to be up-regulated, while another 17 pro-apoptotic genes were found to be repressed (Fig. 2a and 2b). Regarding the anti-apoptotic genes, miR$34 a$ increased the expression level of 10 genes, although it down-regulated the expression of 8 genes (Fig. 2c and 2d). miR-34a did not show a significant impact on the expression of the 23 remaining genes. Our results showed that miR-34a induced the expression of key players involved in the activation of the intrinsic apoptotic pathway, such as BAK (Fig. 2a). BAK triggers mitochondrial outer membrane permeabilization, which leads to the release of cytochrome $C$ into the cytoplasm. Then, APAF1 and cytochrome $\mathrm{C}$ form the apoptosome, which activates caspase- 9 ultimately resulting in apoptotic cell death. ${ }^{19}$ In our study, miR-34a was found to not only increase BAK expression but also to induce the expression of the downstream mediators of intrinsic apoptosis cytochrome C, APAF1 and caspase-9 (Fig. 2a).

PCR array revealed that miR-34a may have also induced extrinsic apoptosis via increasing the levels of TNF signaling pathway mediators (Fig. 2a). TNF-alpha leads to apoptosis via binding to the TNF receptor (TNFR), which in turn activates the Fas-associated protein with death domain (FADD), which then binds to caspase- 8 to form the apoptotic death-inducing signaling complex (DISC). ${ }^{20,21}$ The findings of the current study indicate that the levels of TNF, TNFR, and caspase-8 are elevated by miR-34a ectopic expression (Fig. 2a).

Induction of both intrinsic and extrinsic apoptotic pathways leads to activation of caspases-3, -6 and -7.22 These executioner caspases mediate the cleavage of several proteins critical to cell survival, including actin, lamin, poly (ADP-ribose) polymerase (PARP) and inhibitor of caspaseactivated DNase (ICAD). Cleavage of these proteins leads to molecular and morphological changes characteristic to apoptosis, including cell shrinking and membrane blebbing, disruption of nuclear membrane integrity, DNA fragmentation and impaired DNA repair. ${ }^{23}$ Executioner caspases-3, -6 and -7 were all found to be induced by miR-34a in the present study (Fig. 2a). This finding corroborates a previous study in which miR-34a ectopic overexpression in Hep3B cells and SNU449 cells was shown to promote apoptosis and induce caspase- 3 and -7 activity. ${ }^{13}$ Nonetheless, these findings challenge previous reports which did not observe any impact on apoptosis upon overexpressing miR-34a in HepG2 cells. ${ }^{5,12}$

Finally, this study aimed to evaluate the net impact of miR$34 a$ on cell viability using MTT assay. Results showed that forcing the expression of miR-34a in Huh-7 cells reduced the cell viability significantly (Fig. 3). This finding is in line with other studies reporting that after forcing miR-34a expression in HCC cells, a decrease in cell viability is observed. ${ }^{13,15}$ In the current study, the decrease in cell viability could be explained by the induction of apoptosis, as revealed by the results of the PCR array. In addition, it is possible that the effect of miR-34a on cell viability could also occur via targeting of cell cycle genes, since previous studies have reported that miR-34a has validated targets involved in cell cycle regulation, including NMYC, ${ }^{2}$ CCND $1,{ }^{3}$ CCNE2, CDK4 and CDK6. ${ }^{4}$ 


\section{Conclusions}

This study provides possible explanations for the controversial findings regarding the expression pattern of miR-34a and its role in apoptosis in HCC. Together with findings from previous reports, the current data suggests that miR-34a expression pattern could be etiology-based and stage-specific, and low expression of miR-34a may predict poor prognosis. In this study, it was shown that miR-34a activates dozens of proapoptotic and anti-apoptotic genes, with a net effect of inducing apoptosis and repressing cell viability and, hence, emphasizing the role of miR-34a in apoptosis.

\section{Conflict of interest}

None

\section{Author contributions}

Contributed in study concept and design, execution of experiments, acquisition of data, statistical analysis and interpretation of data, and writing of the manuscript (RY), assisted in experiments, interpretation of data, and revision of the manuscript (IF), assisted in experiments and interpretation of data (RA), provided tissue samples and clinical data $(\mathrm{KH}, \mathrm{GE})$, performed PCR array experiment and contributed in critical revision of the manuscript (ARZ), contributed reagents (HET), contributed in study concept and design and study supervision and provided critical revision of the manuscript for important intellectual content (AIA).

\section{References}

[1] Bommer GT, Gerin I, Feng Y, Kaczorowski AJ, Kuick R, Love RE, et al. p53mediated activation of miRNA34 candidate tumor-suppressor genes. Curr Biol 2007;17:1298-1307. doi: 10.1016/j.cub.2007.06.068.

[2] Cole KA, Attiyeh EF, Mosse YP, Laquaglia MJ, Diskin SJ, Brodeur GM, et al. A functional screen identifies miR-34a as a candidate neuroblastoma tumor suppressor gene. Mol Cancer Res 2008;6:735-742. doi: 10.1158/15417786. MCR-07-2102.

[3] Fujita Y, Kojima K, Hamada N, Ohhashi R, Akao Y, Nozawa Y, et al. Effects of miR-34a on cell growth and chemoresistance in prostate cancer PC3 cells. Biochem Biophys Res Commun 2008;377:114-119. doi: 10.1016/j.bbrc. 2008.09.086.

[4] Toyota M, Suzuki H, Sasaki Y, Maruyama R, Imai K, Shinomura $Y$, et al. Epigenetic silencing of microRNA-34b/c and B-cell translocation gene 4 is associated with CpG island methylation in colorectal cancer. Cancer Res 2008;68:4123-4132. doi: 10.1158/0008-5472.CAN-08-0325.

[5] Li N, Fu H, Tie Y, Hu Z, Kong W, Wu Y, et al. miR-34a inhibits migration and invasion by down-regulation of $\mathrm{c}$-Met expression in human hepatocellular carcinoma cells. Cancer Lett 2009;275:44-53. doi: 10.1016/j.canlet. 2008. 09.035 .
[6] Tarasov V, Jung P, Verdoodt B, Lodygin D, Epanchintsev A, Menssen A, et al. Differential regulation of microRNAs by $\mathrm{p} 53$ revealed by massively parallel sequencing: miR-34a is a p53 target that induces apoptosis and G1-arrest. Cell Cycle 2007;6:1586-1593. doi: 10.4161/cc.6.13.4436

[7] Tazawa H, Tsuchiya N, Izumiya M, Nakagama H. Tumor-suppressive miR-34a induces senescence-like growth arrest through modulation of the E2F pathway in human colon cancer cells. Proc Natl Acad Sci U S A 2007;104: 15472-15477. doi: 10.1073/pnas.0707351104.

[8] Yan D, Zhou X, Chen X, Hu DN, Dong XD, Wang J, et al. MicroRNA-34a inhibits uveal melanoma cell proliferation and migration through downregulation of c-Met. Invest Ophthalmol Vis Sci 2009;50:1559-1565. doi: 10.1167/iovs. 08-2681.

[9] Chen QR, Yu LR, Tsang P, Wei JS, Song YK, Cheuk A, et al. Systematic proteome analysis identifies transcription factor YY1 as a direct target of miR-34a. J Proteome Res 2011;10:479-487. doi: 10.1021/pr1006697.

[10] Welch C, Chen Y, Stallings RL. MicroRNA-34a functions as a potential tumor suppressor by inducing apoptosis in neuroblastoma cells. Oncogene 2007; 26:5017-5022. doi: 10.1038/sj.onc.1210293.

[11] Li L, Yuan L, Luo J, Gao J, Guo J, Xie X. MiR-34a inhibits proliferation and migration of breast cancer through down-regulation of Bcl-2 and SIRT1. Clin Exp Med 2013;13:109-117. doi: 10.1007/s10238-012-0186-5.

[12] Cheng J, Zhou L, Xie QF, Xie HY, Wei XY, Gao F, et al. The impact of miR-34a on protein output in hepatocellular carcinoma HepG2 cells. Proteomics 2010; 10:1557-1572. doi: 10.1002/pmic.200900646.

[13] Dang $Y$, Luo D, Rong $M$, Chen G. Underexpression of miR-34a in hepatocellular carcinoma and its contribution towards enhancement of proliferating inhibitory effects of agents targeting c-MET. PLoS One 2013;8:e61054. doi: 10.1371/journal.pone.0061054.

[14] Pok S, Wen V, Shackel N, Alsop A, Pyakurel P, Fahrer A, et al. Cyclin E facilitates dysplastic hepatocytes to bypass $\mathrm{G} 1 / \mathrm{S}$ checkpoint in hepatocarcinogenesis. J Gastroenterol Hepatol 2013;28:1545-1554. doi: 10.1111/jgh. 12216.

[15] Lou W, Chen Q, Ma L, Liu J, Yang Z, Shen J, et al. Oncolytic adenovirus coexpressing miRNA-34a and IL-24 induces superior antitumor activity in experimental tumor model. J Mol Med (Berl) 2013;91:715-725. doi: 10. 1007/s00109-012-0985-x

[16] Bader AG. miR-34 - a microRNA replacement therapy is headed to the clinic. Front Genet 2012;3:120. doi: 10.3389/fgene.2012.00120.

[17] Pineau P, Volinia S, McJunkin K, Marchio A, Battiston C, Terris B, et al. miR221 overexpression contributes to liver tumorigenesis. Proc Natl Acad Sci U S A 2010;107:264-269. doi: 10.1073/pnas.0907904107.

[18] Yang F, Li QJ, Gong ZB, Zhou L, You N, Wang S, et al. MicroRNA-34a Targets $\mathrm{Bcl}-2$ and Sensitizes Human Hepatocellular Carcinoma Cells to Sorafenib Treatment. Technol Cancer Res Treat 2014;13:77-86. doi: 10.7785/tcrt. 2012.500364.

[19] Elmore S. Apoptosis: a review of programmed cell death. Toxicol Pathol 2007;35:495-516. doi: 10.1080/01926230701320337.

[20] Kelliher MA, Grimm S, Ishida Y, Kuo F, Stanger BZ, Leder P. The death domain kinase RIP mediates the TNF-induced NF-kappaB signal. Immunity 1998;8: 297-303. doi: 10.1016/S1074-7613(00)80535-X.

[21] Kischkel FC, Hellbardt S, Behrmann I, Germer M, Pawlita M, Krammer PH, et al. Cytotoxicity-dependent APO-1 (Fas/CD95)-associated proteins form a death-inducing signaling complex (DISC) with the receptor. EMBO J 1995; 14:5579-5588.

[22] Slee EA, Adrain C, Martin SJ. Executioner caspase-3, -6, and -7 perform distinct, non-redundant roles during the demolition phase of apoptosis. J Biol Chem 2001;276:7320-7326. doi: 10.1074/jbc.M008363200.

[23] Sakahira H, Enari M, Nagata S. Cleavage of CAD inhibitor in CAD activation and DNA degradation during apoptosis. Nature 1998;391:96-99. doi: 10. $1038 / 34214$. 